\title{
Regresión con variables ortogonales y regresión alzada en el modelo STIRPAT
}

\author{
Claudia García García ${ }^{a}$, Catalina B. García García ${ }^{a}$, Román \\ SALMERÓN GÓMEZ ${ }^{a}$, JosÉ GARCíA PÉREZ ${ }^{b}$ \\ a Universidad de Granada, Facultad de Ciencias Económicas y Empresariales, Campus de Cartuja, s/n, 18071 \\ Granada,España.E-mail: claudiagarciagarcia93@gmail.com, cbgarcia@ugr.es, romansg@ugr.es \\ b Universidad de Almería, Edificio Departamental de Ciencias Económicas y Empresariales, Ctra. \\ Sacramento s/n, La Cañada, 04120 Almería. E-mail: jgarcia@ual.es
}

\begin{abstract}
RESUMEN
El modelo STIRPAT representa una de las metodologías más aplicadas en los trabajos sobre medio ambiente. La propia especificación de este modelo econométrico conlleva problemas de multicolinealidad. Aunque una primera opción podría ser eliminar la(s) variable(s) que genera(n) la colinealidad, esto no nos permite estimar los efectos de las consideradas principales fuerzas que provocan impactos medioambientales. Por este motivo, es necesario desarrollar o aplicar nuevos métodos que puedan mitigar este problema de colinealidad en el modelo SITRPAT. Dentro de este marco, se presentan dos regresiones alternativas a la estimación tradicional por Mínimos Cuadrados Ordinarios (MCO): la regresión alzada y la regresión con variables ortogonales. Ambas metodologías consiguen mitigar la colinealidad entre variables existente en el modelo original y, además, nos muestran dos perspectivas diferentes de las variables: mientras el método alzado se aplica desde un punto de vista geométrico, la aplicación de la regresión con variables ortogonales muestra nuevas interpretaciones de las variables.
\end{abstract}

Palabras clave: STIRPAT, Colinealidad, Regresión Alzada, Regresión con Variables Ortogonales.

\section{The Regression with Orthogonal Variables and the Raise Regression in the STIRPAT Model}

\begin{abstract}
STIRPAT model is one of the most analyzed methodologies in environmental studies. The specification of this econometric model provokes multicollinearity. Although a first option could be to eliminate the variable (or variables) that generates the collinearity, it does not allow to estimate the effects of the main forces driving environmental impacts. It is necessary to develop or to apply new methods that could mitigate the collinearity problem in the STIRPAT model. This work applies two methodologies alternatives to the traditional Ordinary Least Squares (OLS) estimation: the raise regression and the regression with orthogonal variables. Both methodologies manage to mitigate the collinearity between variables that exists in the original model, and furthermore, they have two different perspectives about the variables: while the raised method has an important geometric factor in its application, the purpose of the regression with orthogonal variables is to give new interpretations of the variables.
\end{abstract}

Keywords: STIRPAT, Collinearity, Raise Regression, Regression With Orthogonal Variables.

Clasificación JEL: C13, C51, O50, Q53, Q54

Artículo recibido en mayo de 2017 y aceptado en julio de 2017

Artículo disponible en versión electrónica en la página www.revista-eea.net, ref. ə-35307

ISSN 1697-5731 (online) - ISSN 1133-3197 (print) 


\section{INTRODUCCIÓN}

Los estudios sobre medio ambiente han tenido una atención cada vez mayor en los últimos veinte-treinta años. En la actualidad, hay numerosos trabajos sobre este tema que tratan de estimar las emisiones a la atmósfera de algún tipo de gas de efecto invernadero (GEI), por parte de un país o de un grupo de países. Con este propósito y, desde una perspectiva metodológica, es posible distinguir entre métodos de descomposición de índices y métodos econométricos. Los métodos de descomposición de índices nos vienen a decir que el impacto ambiental se puede descomponer en una serie de factores. Por su parte, los métodos econométricos pueden ser usados para realizar test de hipótesis.

Dentro de los métodos de descomposición cabe destacar la identidad IPAT. Esta identidad surge del debate Ehrlich-Holdren/Commoner en los años 70 (Ehrlich y Holdren, 1970, 1971, 1972; Commoner y et al., 1971) sobre las fuerzas antropogénicas que influyen en el impacto medioambiental, tal y como nos dicen York et al. (2003). Lo que nos viene a decir esta identidad es que el impacto ambiental se puede descomponer en tres factores: población, afluencia (PIB per cápita) y un factor tecnológico. Una vez definida esta identidad, a partir de ella se han generado algunas versiones. Las más conocidas son la IPBAT (Schulze, 2002), que incluye además de los factores antes mencionados, el comportamiento de las personas (behavior), y la ImPACT (Waggoner y Ausubel, 2002), que lo que hace es desagregar el factor tecnología en dos factores: el consumo energético y la mejora tecnológica.

Las principales limitaciones que tiene la identidad IPAT es que el número de factores es limitado y además el impacto de los factores es proporcional y se considera que todos los factores afectan en la misma medida al medio ambiente. Además, no permite hacer test de hipótesis. Por estos problemas, surge el modelo STIRPAT, que no es más que la versión estocástica de la identidad IPAT (Dietz y Rosa, 1994; Rosa y Dietz, 1998). Este modelo STIRPAT ya no pertenece a los métodos de descomposición, sino que se incluye en los métodos econométricos.

La especificación del modelo STIRPAT es la siguiente:

$$
I_{i}=a \cdot P_{i}^{b} \cdot A_{i}^{c} \cdot T_{i}^{d} \cdot e_{i}
$$

donde $i$ indica diferentes individuos, $e$ es el término de error, $a$ es la constante del modelo, y $b, c$ y $d$ son los exponentes de las variables $P, A$ y $T$, respectivamente, que han de ser estimados.

Otra forma de expresar el STIRPAT es de la siguiente manera:

$$
\ln \left(I_{i}\right)=a+b \ln \left(P_{i}\right)+c \ln \left(A_{i}\right)+d \ln \left(T_{i}\right)+e_{i}
$$

En este modelo, se puede considerar que el factor $T$ es un factor problemático por los siguientes motivos: 
1) Su especificación:

- En numerosos estudios, las variables $I, P$ y $A$ se utilizan para calcular $T$ (e.g., Ehrlich y Holdren, 1972; Raskin, 1995). Como para la afluencia $(A)$ se toma el PIB per cápita, entonces $P \cdot A=P \cdot(P I B / P)=P I B$. Por definición, $T=I /(P \cdot A)$, y con lo anterior esto se puede escribir también como $T=I / P I B$.

- En otros estudios, el factor $T$ representa una variable independiente del resto de factores, como por ejemplo la formación de capital fijo (e.g., Disli et al.,, 2016) o la actividad del sector industrial (e.g., Gassebner et al., 2011).

2) Es un factor que se suele descomponer en más variables, como es el caso de este trabajo que parte de la versión estocástica de la identidad ImPACT y no de la IPAT.

3) Pueden aparecer problemas de colinealidad en el modelo provocados por este factor, generalmente por su descomposición en más variables (e.g., Harbaugh et al., 2002; Fan et al., 2006; Martínez-Zarzoso et al., 2007; Jia et al., 2009; Lin et al., 2009; Büchs y Schnepf, 2013; Uddin et al., 2016; Dong et al., 2017).

Centrándonos en el tercero de los problemas, una de las soluciones que se toman bajo existencia de colinealidad en un modelo econométrico es eliminar la variable que es el origen de dicho problema. Con este trabajo se trata de demostrar que no es necesario eliminar ninguna de las variables del modelo original. En vez de utilizar la metodología tradicional de Mínimos Cuadrados Ordinarios (MCO), basta con utilizar otros métodos que nos permitan mitigar el problema de la colinealidad entre variables.

Aunque, como acabamos de decir, la existencia de multicolinealidad en el modelo STIRPAT es un problema inherente al mismo, no se ha tratado en muchos estudios de este tipo. Generalmente, lo que se ha hecho es aplicar Mínimos Cuadrados Parciales (e.g., Fan et al., 2006; Jia et al., 2009) o utilizar estimadores cresta (e.g., Lin et al., 2009; Uddin et al., 2016; Dong et al., 2017), sin demostrar al final si se consigue mejorar el modelo. Con este trabajo se propondrán dos métodos alternativos a los utilizados tradicionalmente (como Mínimos Cuadrados Parciales o estimadores cresta): la regresión con variables alzadas y la regresión con variables ortogonales. Estos métodos nos permiten mitigar la multicolinealidad en el modelo original sin tener que eliminar ninguna variable y demostrando al final que se consiguen mejorar los resultados.

En la siguiente sección se analizará la metodología que hay detrás de dichos tipos de regresión y en la sección 3 se aplicarán éstos al modelo STIRPAT, 
examinando los resultados obtenidos. Finalmente, en la última sección se comentarán las conclusiones a las que hemos llegado.

\section{METODOLOGÍA}

Como ya sabemos, en presencia de multicolinealidad en un modelo econométrico existirá una alta inestabilidad de los resultados obtenidos, es decir, con pequeños cambios en las variables explicativas tendremos estimaciones muy diferentes, y probablemente los regresores estimados tendrán además una varianza muestral alta. Por tanto, el primer paso que debemos seguir es detectar la presencia de este problema en el modelo que especifiquemos.

La colinealidad entre variables explicativas puede detectarse utilizando numerosos métodos. En este trabajo, el problema se detectará a través de los siguientes indicadores:

- Determinante de la matriz de correlación: si el valor de este determinante es próximo a cero, entonces podemos decir que tenemos problemas de multicolinealidad en nuestro modelo. Si por el contrario es próximo a uno, no tendremos problemas.

- Número de condición (NC): este valor está basado en los autovalores de la matriz de variables explicativas, $X$,

$$
N C=\sqrt{\frac{\mu_{\max }}{\mu_{\min }}}
$$

donde $\mu$ representa los autovalores de la matriz X. El valor del NC es mayor a uno, y Belsley et al. (1980) nos dicen que valores de este número menores a 20 implican baja multicolinealidad, valores entre 20 y 30 suponen colinealidad moderada, y si el NC es mayor a 30 entonces estaremos ante problemas de multicolinealidad graves.

- Factor de Inflación de la Varianza (FIV): cada una de nuestras variables independientes tiene asociado un FIV,

$$
\mathrm{FIV}_{k}=\frac{1}{1-\mathrm{R}_{k}^{2}}
$$

donde $\mathrm{R}_{k}^{2}$ es el coeficiente determinación de la regresión de la variable $\mathrm{k}$ en función del resto de variables explicativas del modelo. Generalmente se considera que existen problemas importantes de multicolinealidad en el modelo si el valor del FIV es mayor a 10 (e.g., Neter et al., 1989; Marquardt, 1970; Kennedy, 1992; Hair et al., 1995). 


\subsection{Regresión con variables ortogonales}

La regresión con variables ortogonales fue presentada por Novales et al. (2015) para el caso de dos variables independientes. Salmerón et al. (2016) la muestran para el caso de tres variables.

Para ver cuál es el funcionamiento de esta metodología, definamos lo primero un modelo lineal sencillo para dos variables explicativas:

$$
\mathrm{y}=\beta_{0}+\beta_{1} \mathrm{x}_{1}+\beta_{2} \mathrm{x}_{2}+u
$$

Supondremos que este modelo presenta problemas de colinealidad entre variables, es decir, las variables $\mathrm{x}_{1} \mathrm{y}_{\mathrm{x}_{2}}$ están relacionadas. El objetivo de la ortogonalización de una de las variables es aislar su efecto de la otra, o del resto de variables independientes si hubiese más de dos.

En este ejemplo sencillo, vamos a ortogonalizar la variable $\mathrm{x}_{1}$. Para ello, definimos la siguiente regresión auxiliar:

$$
\mathrm{x}_{1}=\alpha_{0}+\alpha_{1} \mathrm{x}_{2}+v
$$

Al estimar esta regresión (6) por MCO, obtendremos los residuos correspondientes, $e_{1}$. Estos residuos representan la parte de la variable $\mathrm{x}_{1}$ que no tiene ningún tipo de relación con la variable $\mathrm{x}_{2}$. Y esto es así por las características de la estimación por MCO: cuando estamos estimando cualquier tipo de regresión por MCO, los residuos estimados serán ortogonales a las variables explicativas de dicho modelo; que los residuos sean ortogonales quiere decir que no compartirán ningún tipo de información con las variables independientes del modelo.

Una vez obtenidos los residuos estimados, $e_{1}$, lo que hacemos ahora es sustituir en el modelo inicial (5) la variable $\mathrm{x}_{1}$ por $e_{1}$. De esta manera, estamos incluyendo solamente una parte de esa variable, y será la parte de $\mathrm{x}_{1}$ que no comparte información con $x_{2}$.

$$
\mathrm{y}=\beta_{0}+\beta_{1} e_{1}+\beta_{2} \mathrm{x}_{2}+u
$$

Por lo tanto, con nuestro modelo final (7), la relación entre las variables desaparecerá y habremos mitigado el problema inicial de multicolinealidad en nuestro modelo (5).

\subsection{Regresión alzada}

La regresión con variables alzadas fue presentada y desarrollada por los trabajos de García et al. (2013, 2017). 
Supongamos que partimos del modelo inicial (5) de la sección anterior que presentaba problemas de colinealidad entre las variables $\mathrm{x}_{1} \quad \mathrm{y} \quad \mathrm{x}_{2}$. Este problema surge porque el vector $\mathrm{x}_{1} \mathrm{y}$ el vector $\mathrm{x}_{2}$ están muy cercanos geométricamente, es decir, el ángulo que forman estos dos vectores $(\theta)$ es muy pequeño. Con la regresión alzada lo que hacemos es separar ambos vectores a través de la regresión auxiliar (6) definida en la sección anterior, cuya estimación por MCO nos lleva a obtener los residuos estimados $e_{1}$. El vector alzado será el siguiente:

$$
\tilde{\mathrm{x}}_{1}=\mathrm{x}_{1}+\lambda e_{1}
$$

Si sustituimos en nuestro modelo (5) la variable $\mathrm{x}_{1}$ por el vector alzado $\tilde{\mathrm{x}}_{1}$ conseguiremos separar las variables geométricamente $\mathrm{y}$, por tanto, el problema de colinealidad se mitigará.

Nuestro modelo final sería por tanto:

$$
\mathrm{y}=\beta_{0}+\beta_{1} \tilde{\mathrm{x}}_{1}+\beta_{2} \mathrm{x}_{2}+u
$$

La Figura 1 representa gráficamente el procedimiento realizado en la regresión alzada.

Figura 1

Regresión alzada

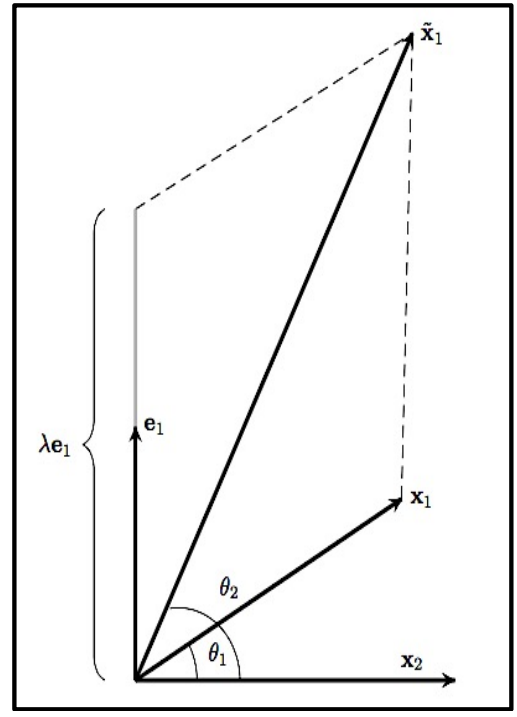

Fuente: Elaboración propia. 


\section{EL MODELO STIRPAT}

Para el análisis empírico de los dos tipos de metodología explicadas anteriormente, se va a usar un conjunto de datos para 78 países durante el año 2013. Todos los datos han sido extraídos de la base de datos del Banco Mundial.

Las variables que se usarán serán las siguientes:

- Impacto ambiental ( $\mathrm{I}$ ): emisiones de dióxido de carbono $\left(\mathrm{CO}_{2}\right)$ per cápita, kt.

- Población ( $P$ ): total de la población de cada país.

- Afluencia (A): PIB per cápita (US\$ corrientes).

- Consumo energético $(C)$ : Uso energético/PIB , donde el uso energético es el uso de energía primaria antes de su transformación (kg de equivalente de petróleo por persona).

- Tecnología ( $T$ ): Emisiones/Uso energético , donde las emisiones están representadas por $I$.

Una vez definidas las variables, pasamos a definir nuestro modelo inicial:

$$
\ln \left(I_{i}\right)=\beta_{0}+\beta_{1} \ln \left(P_{i}\right)+\beta_{2} \ln \left(A_{i}\right)+\beta_{3} \ln \left(C_{i}\right)+\beta_{4} \ln \left(T_{i}\right)+u_{i}
$$

En este modelo, se ha desagregado el factor $T$ en dos variables como hacía la identidad ImPACT. La razón de utilizar dos variables en vez de una es ver la aplicación de la ortogonalización y del alzamiento de una segunda variable, además de que desde un punto de vista empírico tendríamos más información sobre las variables que influyen en el impacto ambiental.

A pesar de que podemos anticipar que las dos variables en las que se ha desagregado el factor $T$ causarán problemas de multicolinealidad en el modelo, a continuación veremos que no es necesario eliminarlas al estar utilizando metodologías alternativas al análisis de regresión tradicional por MCO.

\subsection{Regresión con variables ortogonales en el modelo STIRPAT}

Para comenzar, veamos nuestro modelo (10) estimado por MCO. Si observamos los resultados de la Tabla 2, podemos ver que como era de esperar las variables que nos están causando mayores problemas de colinealidad son las correspondientes al consumo energético ( $C$ ) y a la tecnología ( $T$ ).

En primer lugar, vamos a definir una primera regresión auxiliar para la variable $C$ ya que es la que mayor FIV presenta.

$$
\ln \left(C_{i}\right)=\alpha_{0}+\alpha_{1} \ln \left(P_{i}\right)+\alpha_{2} \ln \left(A_{i}\right)+\alpha_{3} \ln \left(T_{i}\right)+v_{i}
$$




\section{Tabla 1}

Resultados de la estimación por MCO de los modelos (10), (12) y (14). Valores de la desviación típica estimada entre paréntesis

\begin{tabular}{|c|c|c|c|}
\hline $\ln \left(\hat{I}_{i}\right)$ & Modelo (10) & Modelo (12) & Modelo (14) \\
\hline Constante & $-0.034(0.087)$ & $-3.011(0.085)$ & $-9.872(0.050)$ \\
\hline $\ln \left(P_{i}\right)$ & $1.005(0.006)$ & $0.525(0.005)$ & $1.031(0.002)$ \\
\hline $\ln (A)$ & $0.999(0.006)$ & $0.408(0.004)$ & $0.433(0.004)$ \\
\hline $\ln \left(C_{i}\right)$ & $1.002(0.007)$ & - & - \\
\hline$e_{i}^{C}$ & - & $1.002(0.007)$ & $1.002(0.007)$ \\
\hline $\ln \left(T_{i}\right)$ & $0.998(0.006)$ & $0.493(0.005)$ & - \\
\hline$e_{i}^{T}$ & - & - & $0.493(0.005)$ \\
\hline $\mathbf{S C R}_{\mathbf{R}^{2}}$ & -3 & 0.0766 \\
\hline $\mathrm{F}_{\exp }$ & \multicolumn{3}{|c}{$\mathrm{F}_{4.73}=97650$} \\
\hline
\end{tabular}

Fuente: Elaboración propia a partir de los datos obtenidos de la base de datos del Banco Mundial.

Estimando por MCO la regresión (11) obtendremos los residuos correspondientes, que denotaremos por $e_{i}^{C}$. Estos residuos sustituirán a la variable $\ln \left(C_{i}\right)$ en el modelo inicial (10), y van a representar la parte de dicha variable que no tiene ningún tipo de relación o que no comparte ninguna información con el resto de variables independientes de nuestro modelo (10).

$$
\ln \left(I_{i}\right)=\beta_{0}+\beta_{1} \ln \left(P_{i}\right)+\beta_{2} \ln \left(A_{i}\right)+\beta_{3} e_{i}^{C}+\beta_{4} \ln \left(T_{i}\right)+u_{i}
$$

En la Tabla 2 podemos observar que atendiendo a los valores del FIV para este nuevo modelo parece que los problemas de colinealidad se han resuelto (los valores para todas las variables del modelo (12) son menores a 10). Sin embargo, el determinante de la matriz de correlación sigue siendo más próximo a 0 que a 1 y el NC sigue teniendo un valor elevado. Esto podría deberse a que la otra variable considerada problemática, $T$, no se ha modificado por lo que nos sigue causando problemas. Por tanto, definimos una segunda regresión auxiliar:

$$
\ln \left(T_{i}\right)=\delta_{0}+\delta_{1} \ln \left(P_{i}\right)+\delta_{2} \ln \left(A_{i}\right)+w_{i}
$$

En esta segunda regresión auxiliar podemos ver que ya no aparece como variable explicativa el consumo energético $(C)$. Como en la regresión (11) hemos incluido el factor $T$, si incluímos $e_{i}^{C}$ en el modelo (10) ya conseguimos 
que el consumo energético sea ortogonal al factor tecnológico, por lo que incluir como variable explicativa $e_{i}^{C}$ en el modelo (13) no aportaría nada.

\section{Tabla 2}

Multicolinealidad en los modelos (10), (12) y (14)

\begin{tabular}{|c|c|c|c|c|}
\hline \multicolumn{2}{|c|}{$\ln \left(\hat{I}_{i}\right)$} & Modelo (10) & Modelo (12) & Modelo (14) \\
\hline \multirow{4}{*}{ FIV $_{\mathbf{k}}$} & $\ln \left(P_{i}\right)$ & 13.035 & 9.676 & 1.006 \\
\cline { 2 - 5 } & $\ln \left(A_{i}\right)$ & 1.916 & 1.009 & 1.006 \\
\cline { 2 - 5 } & $\ln \left(C_{i}\right)$ & 16.382 & - & - \\
\cline { 2 - 5 } & $e_{i}^{C}$ & - & 1.000 & 1.000 \\
\cline { 2 - 5 } & $\ln \left(T_{i}\right)$ & 14.002 & 9.647 & - \\
\cline { 2 - 5 } & $e_{i}^{T}$ & - & - & 1.000 \\
\hline \multicolumn{2}{|c|}{$\begin{array}{c}\text { Determinante matriz } \\
\text { correlación }\end{array}$} & 0.006 & 62.141 & 0.994 \\
\hline
\end{tabular}

Fuente: Elaboración propia a partir de los datos obtenidos de la base de datos del Banco Mundial.

Estimando por MCO el modelo (13) obtendremos los residuos correspondientes, que denotaremos por $e_{i}^{T}$, y que sustituirán en el modelo (12) a la variable $\ln \left(T_{i}\right)$.

$$
\ln \left(I_{i}\right)=\beta_{0}+\beta_{1} \ln \left(P_{i}\right)+\beta_{2} \ln \left(A_{i}\right)+\beta_{3} e_{i}^{C}+\beta_{4} e_{i}^{T}+u_{i}
$$

Los resultados de la estimación por MCO de los tres modelos (10), (12) y (14), se pueden observar en las Tablas 1 y 2.

Observando los resultados de nuestro modelo final (14), podemos afirmar que: todos nuestros estimadores son significativos de forma individual, por lo que podemos decir que nuestras variables independientes son importantes a la hora de explicar el impacto ambiental. Si analizamos los valores obtenidos en la estimación (ver Tabla 1):

- Cuando aumenta un 1\% la población de un país, el impacto ambiental, medido en emisiones de $\mathrm{CO}_{2}$ per cápita, aumentará en un 1.031\%. En otras palabras, las emisiones de un país aumentan a la misma velocidad que la población.

- Si observamos la variable relativa al PIB per cápita, la afluencia, podemos decir que cuando ésta aumenta en un 1\%, las emisiones a la atmósfera 
aumentan en un $0.433 \%$. Es decir, el impacto ambiental aumenta aproximadamente a la mitad de velocidad que lo hace el PIB per cápita.

- Cuando la parte del consumo energético que no tiene ninguna relación con el resto de variables de nuestro modelo, $e_{i}^{C}$, aumenta en un $1 \%$, las emisiones de $\mathrm{CO}_{2}$ per cápita aumentarán un $1.002 \%$. Por tanto, estas variables aumentan a la misma velocidad.

- Si aumenta el factor tecnológico (su parte no relacionada con el resto de variables, $e_{i}^{T}$ ) en un $1 \%$, el impacto ambiental lo hará en un $0.493 \%$. Lo que estamos diciendo con esto es que un empeoramiento tecnológico hace que el impacto ambiental aumente: un aumento del factor $T$ nos está diciendo que aumentarán las emisiones emitidas por kg de energía utilizada, por lo que la tecnología es peor.

Además nuestro modelo es bueno de forma global: conseguimos explicar casi el $100 \%$ de la variabilidad de los datos y es significativo globalmente.

Finalmente, respecto a los problemas de multicolinealidad existentes en el modelo inicial (10), podemos decir que aplicando la regresión con variables ortogonales conseguimos mitigar el problema: los valores del FIV son mínimos, el determinante de la matriz de correlación tiene un valor próximo a 1 y el NC es menor a 30 .

Antes de pasar al siguiente método, es importante decir que la regresión ortogonal ha tenido sentido desde un punto de vista "interpretativo" de las variables, es decir, ha tenido sentido aislar el efecto de las variables que se han ortogonalizado. Es útil ortogonalizar las dos variables escogidas, no solo por el hecho de que son las que provocan los principales problemas de multicolinealidad en el modelo, sino porque desde un punto de vista de su interpretación es interesante aislar su efecto:

- La variable $C$ representa el consumo de energía en relación a la productividad del país. Aislando el efecto de esta variable, se puede observar la eficiencia energética real de cada país, independientemente de su estructura económica, su población y su nivel tecnológico.

- La variable $T$ es una medida del nivel tecnológico de un país. Si su valor es bajo, estaremos diciendo que el país es menos contaminante en relación a la energía que utiliza. Si aislamos su efecto individual, podemos observar la eficiencia ecológica real del país, independientemente de su estructura económica, de su población y de su eficiencia energética.

\subsection{Regresión alzada en el modelo STIRPAT}

En la regresión alzada, debemos especificar cuál es la variable sustituta en nuestro modelo inicial. 
Partiendo del modelo inicial (10), en primer lugar vamos a definir la variable alzada para el consumo energético $(C)$. Para ello, utilizando los residuos de la estimación del modelo (11), que habíamos denominado como $e_{i}^{C}$, definimos la siguiente variable:

$$
\tilde{C}_{i}=\ln \left(C_{i}\right)+\lambda e_{i}^{C}
$$

Se han dado diferentes valores para $\lambda(\lambda>0)$, y con los diferentes resultados obtenidos para la variable $\tilde{C}_{i}$, se ha estimado el siguiente modelo por MCO:

$$
\ln \left(I_{i}\right)=\beta_{0}+\beta_{1} \ln \left(P_{i}\right)+\beta_{2} \ln \left(A_{i}\right)+\beta_{3} \tilde{C}_{i}+\beta_{4} \ln \left(T_{i}\right)+u_{i}
$$

Los resultados obtenidos en la estimación MCO del modelo (16) para distintos valores de $\lambda$ se pueden observar en las Tablas 3 y 4 .

Tabla 3

\begin{tabular}{|c|c|c|}
\hline$\lambda$ & $\hat{\beta}_{k}$ & d.t.k \\
\hline 0 & $(-0.034 ; 1.005 ; 0.999 ; 1.002 ; 0.998)$ & $(0.087 ; 0.006 ; 0.006 ; 0.007 ; 0.006)$ \\
\hline 0.1 & $(-0.305 ; 0.961 ; 0.944 ; 0.911 ; 0.952)$ & $(0.087 ; 0.006 ; 0.005 ; 0.006 ; 0.006)$ \\
\hline 0.2 & $(-0.530 ; 0.925 ; 0.900 ; 0.835 ; 0.914)$ & $(0.087 ; 0.006 ; 0.005 ; 0.005 ; 0.006)$ \\
\hline 0.3 & $(-0.721 ; 0.894 ; 0.863 ; 0.771 ; 0.882)$ & $(0.087 ; 0.006 ; 0.005 ; 0.005 ; 0.006)$ \\
\hline 0.4 & $(-0.884 ; 0.868 ; 0.830 ; 0.716 ; 0.854)$ & $(0.086 ; 0.006 ; 0.005 ; 0.005 ; 0.005)$ \\
\hline 0.5 & $(-1.026 ; 0.845 ; 0.802 ; 0.668 ; 0.830)$ & $(0.086 ; 0.006 ; 0.005 ; 0.004 ; 0.005)$ \\
\hline 0.6 & $(-1.150 ; 0.825 ; 0.777 ; 0.626 ; 0.809)$ & $(0.086 ; 0.006 ; 0.005 ; 0.004 ; 0.005)$ \\
\hline 0.7 & $(-1.260 ; 0.807 ; 0.756 ; 0.589 ; 0.790)$ & $(0.086 ; 0.006 ; 0.005 ; 0.004 ; 0.005)$ \\
\hline 0.8 & $(-1.357 ; 0.792 ; 0.736 ; 0.557 ; 0.774)$ & $(0.086 ; 0.006 ; 0.005 ; 0.004 ; 0.005)$ \\
\hline 0.9 & $(-1.444 ; 0.777 ; 0.719 ; 0.527 ; 0.759)$ & $(0.086 ; 0.006 ; 0.005 ; 0.003 ; 0.005)$ \\
\hline 1 & $(-1.522 ; 0.765 ; 0.704 ; 0.501 ; 0.746)$ & $(0.086 ; 0.006 ; 0.004 ; 0.003 ; 0.005)$ \\
\hline 2.511 & $(-2, .163 ; 0.662 ; 0.577 ; 0.285 ; 0.637)$ & $(0.085 ; 0.005 ; 0.004 ; 0.002 ; 0.005)$ \\
\hline 5 & $(-2.515 ; 0.605 ; 0.507 ; 0.167 ; 0.577)$ & $(0.085 ; 0.005 ; 0.004 ; 0.001 ; 0.005)$ \\
\hline 10 & $(-2.740 ; 0.569 ; 0.462 ; 0.091 ; 0.539)$ & $(0.085 ; 0.005 ; 0.004 ; 0.001 ; 0.005)$ \\
\hline 15 & $(-2.825 ; 0.555 ; 0.445 ; 0.063 ; 0.525)$ & $(0.085 ; 0.005 ; 0.004 ; 0.000 ; 0.005)$ \\
\hline 20 & $(-2.869 ; 0.548 ; 0.436 ; 0.048 ; 0.517)$ & $(0.085 ; 0.005 ; 0.004 ; 0.000 ; 0.005)$ \\
\hline 25 & $(-2.896 ; 0.544 ; 0.431 ; 0.038 ; 0.513)$ & $(0.085 ; 0.005 ; 0.004 ; 0.000 ; 0.005)$ \\
\hline 50 & $(-2.952 ; 0.535 ; 0.420 ; 0.020 ; 0.503)$ & $(0.085 ; 0.005 ; 0.004 ; 0.000 ; 0.005)$ \\
\hline 100 & $(-2.981 ; 0.530 ; 0.414 ; 0.010 ; 0.498)$ & $(0.085 ; 0.005 ; 0.004 ; 0.000 ; 0.005)$ \\
\hline 200 & $(-2.996 ; 0.528 ; 0.411 ; 0.005 ; 0.496)$ & $(0.085 ; 0.005 ; 0.004 ; 0.000 ; 0.005)$ \\
\hline$\ldots$ & $\ldots$ & $\ldots$ \\
\hline SCR & \multicolumn{2}{|c|}{$\frac{1}{0.0766}$} \\
\hline $\mathbf{R}^{2}$ & \multicolumn{2}{|c|}{0.9998} \\
\hline$F_{\text {exp }}$ & \multicolumn{2}{|c|}{$F_{4,73}=97650$} \\
\hline
\end{tabular}

Resultados de la estimación por MCO del modelo (16)

Fuente: Elaboración propia a partir de los datos obtenidos de la base de datos del Banco Mundial.

Observando la Tabla 4, podemos ver que los problemas de multicolinealidad desde la perspectiva del FIV dejan de ser preocupantes para $\lambda \geq 2.511$. Sin 
embargo, no es así si observamos el NC o el determinante de la matriz de correlación. Tal y como hemos hecho con el método de ortogonalización de variables, vamos a alzar también la variable $T$. Para ello, procedemos como con la variable correspondiente al consumo energético; definimos la variable alzada a partir de la siguiente regresión auxiliar:

$$
\ln \left(T_{i}\right)=\delta_{0}+\delta_{1} \ln \left(P_{i}\right)+\delta_{2} \ln \left(A_{i}\right)+\delta_{3} \tilde{C}_{i}+w_{i}
$$

De manera que la variable alzada, utilizando los residuos de la estimación por MCO de este modelo (17), sería:

$$
\tilde{T}_{i}=\ln \left(T_{i}\right)+\lambda e_{i}^{T}
$$

Y dando diferentes valores a $\lambda(\lambda>0)$, obtendremos distintas estimaciones para el siguiente modelo:

$$
\ln \left(I_{i}\right)=\beta_{0}+\beta_{1} \ln \left(P_{i}\right)+\beta_{2} \ln \left(A_{i}\right)+\beta_{3} \tilde{C}_{i}+\beta_{4} \tilde{T}_{i}+u_{i}
$$

Tabla 4

Multicolinealidad en el modelo (16)

\begin{tabular}{|c|c|c|c|}
\hline$\lambda$ & FIV $_{\mathbf{k}}$ & NC & Determinante matriz correlación \\
\hline $\mathbf{0}$ & $(13.035 ; 1.916 ; 16.382 ; 14.002)$ & 82.709 & 0.006 \\
\hline $\mathbf{0 . 1}$ & $(12.452 ; 1.758 ; 13.712 ; 13.246)$ & 77.360 & 0.007 \\
\hline $\mathbf{0 . 2}$ & $(12.008 ; 1.639 ; 11.682 ; 12.671)$ & 74.258 & 0.009 \\
\hline $\mathbf{0 . 3}$ & $(11.663 ; 1.546 ; 10.102 ; 12.224)$ & 72.682 & 0.010 \\
\hline $\mathbf{0 . 4}$ & $(11.389 ; 1.472 ; 8.848 ; 11.869)$ & 71.860 & 0.012 \\
\hline $\mathbf{0 . 5}$ & $(11.169 ; 1.412 ; 7.836 ; 11.582)$ & 71.387 & 0.013 \\
\hline $\mathbf{0 . 6}$ & $(10.988 ; 1.363 ; 7.009 ; 11.348)$ & 71.088 & 0.015 \\
\hline $\mathbf{0 . 7}$ & $(10.838 ; 1.323 ; 6.322 ; 11.154)$ & 70.885 & 0.016 \\
\hline $\mathbf{0 . 8}$ & $(10.712 ; 1.289 ; 5.747 ; 10.991)$ & 70.739 & 0.018 \\
\hline $\mathbf{0 . 9}$ & $(10.606 ; 1.260 ; 5.261 ; 10.853)$ & 70.630 & 0.020 \\
\hline $\mathbf{1}$ & $(10.515 ; 1.236 ; 4.845 ; 10.735)$ & 70.545 & 0.021 \\
\hline $\mathbf{2 . 5 1 1}$ & $(9.948 ; 1.083 ; 2.248 ; 9.9999)$ & 70.116 & 0.046 \\
\hline $\mathbf{5}$ & $(9.769 ; 1.035 ; 1.427 ; 9.768)$ & 69.897 & 0.072 \\
\hline $\mathbf{1 0}$ & $(9.703 ; 1.017 ; 1.127 ; 9.683)$ & 69.433 & 0.091 \\
\hline $\mathbf{1 5}$ & $(9.689 ; 1.013 ; 1.060 ; 9.663)$ & 68.833 & 0.097 \\
\hline $\mathbf{2 0}$ & $(9.683 ; 1.011 ; 1.035 ; 9.656)$ & 68.163 & 0.100 \\
\hline $\mathbf{2 5}$ & $(9.681 ; 1.011 ; 1.023 ; 9.653)$ & 67.491 & 0.101 \\
\hline $\mathbf{5 0}$ & $(9.677 ; 1.020 ; 1.006 ; 9.648)$ & 64.967 & 0.102 \\
\hline $\mathbf{1 0 0}$ & $(9.676 ; 1.009 ; 1.001 ; 9.647)$ & 63.140 & 0.103 \\
\hline $\mathbf{2 0 0}$ & $(9.676 ; 1.009 ; 1.000 ; 9.647)$ & 62.422 & 0.103 \\
\hline$\ldots$ & & $\ldots$ & $\ldots$ \\
\hline
\end{tabular}

Fuente: Elaboración propia a partir de los datos obtenidos de la base de datos del Banco Mundial.

Los resultados de este modelo (19) se pueden observar en las Tablas 5 y 6 . 
Tabla 5

Resultados de la estimación por MCO del modelo (19)

\begin{tabular}{|c|c|c|}
\hline$\lambda$ & $\hat{\beta}_{k}$ & d.t. . \\
\hline 0 & $(-0.034 ; 1.005 ; 0.999 ; 1.002 ; 0.998)$ & $(0.087 ; 0.006 ; 0.006 ; 0.007 ; 0.006)$ \\
\hline 0.1 & $(-1.320 ; 1.004 ; 0.921 ; 0.864 ; 0.866)$ & $(0.082 ; 0.006 ; 0.005 ; 0.006 ; 0.005)$ \\
\hline 0.2 & $(-2.358 ; 1.009 ; 0.864 ; 0.763 ; 0.762)$ & $(0.078 ; 0.006 ; 0.005 ; 0.005 ; 0.005)$ \\
\hline 0.3 & $(-3.208 ; 1.018 ; 0.820 ; 0.686 ; 0.678)$ & $(0.075 ; 0.005 ; 0.005 ; 0.005 ; 0.004)$ \\
\hline 0.31 & $(-3.284 ; 1.019 ; 0.817 ; 0.679 ; 0.671)$ & $(0.074 ; 0.005 ; 0.005 ; 0.005 ; 0.004)$ \\
\hline 0.4 & $(-3.913 ; 1.028 ; 0.787 ; 0.625 ; 0.610)$ & $(0.072 ; 0.005 ; 0.005 ; 0.004 ; 0.004)$ \\
\hline 0.5 & $(-4.504 ; 1.037 ; 0.759 ; 0.576 ; 0.553)$ & $(0.070 ; 0.005 ; 0.005 ; 0.004 ; 0.004)$ \\
\hline 0.6 & $(-5.006 ; 1.046 ; 0.737 ; 0.536 ; 0.506)$ & $(0.068 ; 0.005 ; 0.005 ; 0.004 ; 0.003)$ \\
\hline 0.7 & $(-5.434 ; 1.054 ; 0.718 ; 0.502 ; 0.465)$ & $(0.066 ; 0.005 ; 0.005 ; 0.004 ; 0.003)$ \\
\hline 0.8 & $(-5.804 ; 1.061 ; 0.702 ; 0.473 ; 0.430)$ & $(0.064 ; 0.004 ; 0.005 ; 0.003 ; 0.003)$ \\
\hline 0.9 & $(-6.126 ; 1.067 ; 0.688 ; 0.448 ; 0.399)$ & $(0.063 ; 0.004 ; 0.005 ; 0.003 ; 0.003)$ \\
\hline 1 & $(-6.407 ; 1.073 ; 0.676 ; 0.426 ; 0.373)$ & $(0.062 ; 0.004 ; 0.004 ; 0.003 ; 0.003)$ \\
\hline 5 & $(-9.161 ; 1.087 ; 0.523 ; 0.155 ; 0.096)$ & $(0.052 ; 0.002 ; 0.004 ; 0.001 ; 0.001)$ \\
\hline 10 & $(-9.544 ; 1.068 ; 0.484 ; 0.087 ; 0.049)$ & $(0.050 ; 0.002 ; 0.004 ; 0.001 ; 0.000)$ \\
\hline 15 & $(-9.662 ; 1.058 ; 0.468 ; 0.061 ; 0.033)$ & $(0.050 ; 0.002 ; 0.004 ; 0.000 ; 0.000)$ \\
\hline 20 & $(-9.718 ; 1.052 ; 0.460 ; 0.047 ; 0.025)$ & $(0.050 ; 0.002 ; 0.004 ; 0.000 ; 0.000)$ \\
\hline 25 & $(-9.751 ; 1.048 ; 0.455 ; 0.038 ; 0.020)$ & $(0.050 ; 0.002 ; 0.004 ; 0.000 ; 0.000)$ \\
\hline 50 & $(-9.814 ; 1.040 ; 0.444 ; 0.019 ; 0.010)$ & $(0.050 ; 0.002 ; 0.004 ; 0.000 ; 0.000)$ \\
\hline 100 & $(-9.843 ; 1.036 ; 0.438 ; 0.010 ; 0.005)$ & $(0.050 ; 0.002 ; 0.004 ; 0.000 ; 0.000)$ \\
\hline 200 & $(-9.858 ; 1.033 ; 0.435 ; 0.005 ; 0.002)$ & $(0.050 ; 0.002 ; 0.004 ; 0.000 ; 0.000)$ \\
\hline$\ldots$ & $\cdots$ & $\cdots$ \\
\hline SCR & \multicolumn{2}{|c|}{0.0766} \\
\hline $\mathrm{R}^{2}$ & \multicolumn{2}{|c|}{0.9998} \\
\hline$F_{\text {exp }}$ & \multicolumn{2}{|c|}{$F_{4,73}=97650$} \\
\hline
\end{tabular}

Fuente: Elaboración propia a partir de los datos obtenidos de la base de datos del Banco Mundial.

Observando los resultados de nuestro modelo final (19) para el mayor valor de $\lambda$ analizado ( $\lambda=200)$, podemos afirmar que: todos nuestros estimadores son significativos de forma individual, por lo que podemos decir que nuestras variables independientes son importantes a la hora de explicar el impacto ambiental. Si analizamos los valores obtenidos en la estimación (ver Tabla 5):

- Cuando aumenta un 1\% la población de un país, el impacto ambiental, medido en emisiones de $\mathrm{CO}_{2}$ per cápita, aumentará en un 1.033\%. En otras palabras, las emisiones de un país aumentan a la misma velocidad que la población.

- Si observamos la variable relativa al PIB per cápita, la afluencia, podemos decir que cuando ésta aumenta en un 1\%, las emisiones a la atmósfera aumentan en un $0.435 \%$. Es decir, el impacto ambiental aumenta aproximadamente a la mitad de velocidad que lo hace el PIB per cápita. 
- Para las variables alzadas, vemos que un aumento de un $1 \%$ en cada una de ellas, el consumo energético y el factor tecnología, hace que aumente muy poco el impacto ambiental: un $0.005 \%$ y un $0.002 \%$, respectivamente.

Tabla 6

Multicolinealidad en el modelo (19)

\begin{tabular}{|c|c|c|c|}
\hline$\lambda$ & FIV $_{\mathbf{k}}$ & NC & Determinante matriz correlación \\
\hline $\mathbf{0}$ & $(13.035 ; 1.916 ; 16.382 ; 14.002)$ & 82.709 & 0.006 \\
\hline $\mathbf{0 . 1}$ & $(11.982 ; 1.730 ; 13.066 ; 11.121)$ & 77.286 & 0.009 \\
\hline $\mathbf{0 . 2}$ & $(10.993 ; 1.604 ; 10.830 ; 9.105)$ & 73.328 & 0.012 \\
\hline $\mathbf{0 . 3}$ & $(10.081 ; 1.513 ; 9.232 ; 7.641)$ & 69.984 & 0.016 \\
\hline $\mathbf{0 . 3 1}$ & $(9.995 ; 1.505 ; 9.097 ; 7.517)$ & 69.672 & 0.017 \\
\hline $\mathbf{0 . 4}$ & $(9.254 ; 1.443 ; 8.037 ; 6.545)$ & 67.002 & 0.021 \\
\hline $\mathbf{0 . 5}$ & $(8.509 ; 1.389 ; 7.109 ; 5.703)$ & 64.296 & 0.028 \\
\hline $\mathbf{0 . 6}$ & $(7.844 ; 1.345 ; 6.368 ; 5.042)$ & 61.826 & 0.033 \\
\hline $\mathbf{0 . 7}$ & $(7.251 ; 1.308 ; 5.764 ; 4.513)$ & 59.572 & 0.040 \\
\hline $\mathbf{0 . 8}$ & $(6.722 ; 1.278 ; 5.261 ; 4.084)$ & 57.516 & 0.048 \\
\hline $\mathbf{0 . 9}$ & $(6.251 ; 1.251 ; 4.838 ; 3.729)$ & 55.643 & 0.057 \\
\hline $\mathbf{1}$ & $(5.830 ; 1.229 ; 4.477 ; 3.434)$ & 53.940 & 0.066 \\
\hline $\mathbf{5}$ & $(1.637 ; 1.033 ; 1.410 ; 1.243)$ & 36.755 & 0.567 \\
\hline $\mathbf{1 0}$ & $(1.196 ; 1.014 ; 1.123 ; 1.072)$ & 34.754 & 0.826 \\
\hline $\mathbf{1 5}$ & $(1.096 ; 1.010 ; 1.058 ; 1.034)$ & 33.958 & 0.909 \\
\hline $\mathbf{2 0}$ & $(1.058 ; 1.008 ; 1.034 ; 1.020)$ & 33.398 & 0.943 \\
\hline $\mathbf{2 5}$ & $(1.040 ; 1.007 ; 1.022 ; 1.013)$ & 32.929 & 0.960 \\
\hline $\mathbf{5 0}$ & $(1.015 ; 1.006 ; 1.006 ; 1.003)$ & 31.350 & 0.985 \\
\hline $\mathbf{1 0 0}$ & $(1.008 ; 1.006 ; 1.001 ; 1.001)$ & 30.218 & 0.992 \\
\hline $\mathbf{2 0 0}$ & $(1.006 ; 1.006 ; 1.000 ; 1.000)$ & 29.760 & 0.994 \\
\hline $\mathbf{2 0}$ & & $\ldots$ & $\ldots$ \\
\hline
\end{tabular}

Fuente: Elaboración propia a partir de los datos obtenidos de la base de datos del Banco Mundial.

Además nuestro modelo es bueno de forma global: conseguimos explicar casi el $100 \%$ de la variabilidad de los datos y es significativo globalmente.

Finalmente, respecto a los problemas de multicolinealidad existentes en el modelo inicial (10), podemos decir que aplicando la regresión con variables alzadas conseguimos mitigar el problema: los valores del FIV son mínimos, el determinante de la matriz de correlación tiene un valor próximo a 1 y el NC es menor a 30 (ver Tabla 6).

\section{CONCLUSIONES}

Para resumir brevemente lo que se ha hecho, en primer lugar se ha explicado cómo aplicar la regresión con variables ortogonales y la regresión alzada. Una vez explicado el funcionamiento básico de estas metodologías, se ha pasado a realizar la aplicación empírica en la estimación del modelo STIRPAT. Para ello, se han utilizado datos de 78 países durante el año 2013. 
En los apartados correspondientes se han analizado los valores de los estimadores para las cuatro variables de nuestro modelo. Es importante recalcar que atendiendo a las variables que no han sido modificadas (población y afluencia), podemos observar que las estimaciones de los parámetros correspondientes son muy similares para ambas metodologías: a medida que en el modelo alzado vamos utilizando valores de $\lambda$ mayores (cuando $\lambda \rightarrow \infty$ ), los resultados cada vez son más parecidos a las estimaciones de la regresión con variables ortogonales.

Analizando la multicolinealidad existente en el modelo STIRPAT, hemos podido ver que la aplicación de ambas metodologías nos permite obtener buenos resultados al mismo tiempo que conseguimos mitigar el problema.

En primer lugar, tanto para la regresión con variables ortogonales como para la regresión alzada, se ha observado el FIV de las variables y se ha detectado que había problemas de colinealidad importantes con las variables correspondientes al consumo energético $(C)$ y a la tecnología $(T)$.

Centrándonos en el primer tipo de metodología analizado, la regresión con variables ortogonales, la primera variable que se ortogonaliza es la correspondiente al consumo, $C$. Esta elección se debe a que es la variable que mayor FIV presenta. Observando la Tabla 2, hemos visto que los problemas de multicolinealidad en el STIRPAT desde la perspectiva del FIV se han mitigado, sin embargo no es así si tenemos en cuenta los resultados para el NC o para el determinante de la matriz de correlación, por lo que se hace necesaria una segunda ortogonalización: la de la variable $T$. Con esta segunda aplicación del método, vemos que sí se consigue mitigar el problema. Esto lo podemos ver no solamente prestando atención a los valores del FIV para cada una de las variables explicativas (que tienen un valor mínimo), sino también observando el NC y el determinante de la matriz de correlación de los regresores. En cuanto al NC vemos que ha disminuido de un valor de 82.709, que representaba la existencia de multicolinealidad grave en el modelo, a un valor de 29.578, que nos muestra que la colinealidad ha pasado a ser moderada. Además, el determinante de la matriz de correlación reafirma la mitigación de la multicolinealidad existente, ya que pasa de tener un valor próximo a 0 a tener un valor próximo a 1 .

Si ahora pasamos a analizar la multicolinealidad del modelo estimado utilizando la metodología de regresión con variables alzadas, vemos que ocurre lo mismo: un primer alzamiento, el de la variable $C$, parece que disminuye la multicolinealidad existente para valores de $\lambda$ mayores a 2.511 desde la perspectiva del FIV (ver Tabla 4); sin embargo, no reduce el NC lo suficiente para decir que se ha mitigado el problema y el determinante de la matriz de correlación sigue siendo próximo a 0 . Por este motivo, hemos alzado también la segunda variable problemática: $T$. Y con esto ya conseguimos mitigar el 
problema de la multicolinealidad (ver Tabla 6): los FIV tienen un valor mínimo, el NC implica la existencia de colinealidad moderada y el valor del determinante de la matriz de correlación es prácticamente 1.

Vemos por tanto, que con la utilización de estas metodologías, tanto la regresión con variables ortogonales como la regresión alzada, conseguimos mitigar el problema de la existencia de multicolinealidad en nuestro modelo de partida, sin tener que eliminar ninguna de las variables de nuestro modelo. Por lo que estaríamos recogiendo más información sobre las variables que afectan al impacto ambiental. Además, hemos partido de un modelo inicial con unas buenas características globales, y la utilización de ambas metodologías nos ha permitido mantenerlas: tanto el $\mathrm{R}^{2}$ como la significación global de nuestro modelo permanecen inalterados.

Para finalizar, la elección del método a aplicar dependerá de la finalidad del trabajo. La regresión con variables ortogonales permite obtener interpretaciones alternativas de los estimadores, y se recomienda su aplicación cuando se desea conocer el efecto individual de las variables. Sin embargo, esta metodología pierde el sentido cuando el efecto aislado de la variable a ortogonalizar no es interpretable. En estos casos, la regresión alzada permite separar geométricamente nuestras variables. Ademas, se ha mostrado que en la regresión alzada para valores altos de $\lambda$ se obtienen resultados similares a la regresión con variables ortogonales para todos los parámetros excepto para los de las variables alzadas.

En conclusión, se han aplicado dos metodologías que mitigan la multicolinealidad en el modelo STIRPAT sin tener que eliminar ninguna variable. En futuros trabajos sería interesante analizar otro tipo de metodologías tradicionalmente utilizadas, como la aplicación de estimadores cresta o de Mínimos Cuadrados Parciales, y comparar estos métodos con los propuestos aquí.

\section{REFERENCIAS BIBLIOGRÁFICAS}

BELSLEY, D.; KUH, E.; WELSCH, R. (1980). Regression diagnostics: Identifying influential data and sources of collinearity. Nueva York: John Wiley and Sons.

BÜCHS, M.; SCHNEPF, S.V. (2013). "Who emits most? Associations between socioeconomic facors and UK households' home energy, transport, indirect and total CO2 emissions". Ecological Economics, 90, pp. 114-123.

COMMONER, B.; CORR, M.; STAMLER, P. (1971). "The causes of pollution". Environment: Science and Policy for Sustainable Development, 13(3), pp. 2-19.

DIETZ, T.; ROSA, E.A. (1994). "Rethinking the environmental impacts of population, affluence and technology". Human Ecology Review, 1, pp. 277-300.

DISLI, M.; NG, A.; ASKARI, H. (2016). "Culture, income, and CO2 emission". Renewable and Sustainable Energy Reviews, 62, pp. 418-428. 
DONG, J.; DENG, C.; LI, R.; HUANG, J. (2017). "Moving Low-Carbon Transportation in Xinjiang: Evidence from STIRPAT and Rigid Regression Models". Sustainability, 9, pp. 24.

EHRLICH, P.R.; HOLDREN, J.P. (1970). "The people problem". Saturday Review, 4(42), pp. $42-43$.

EHRLICH, P.R.; HOLDREN, J.P. (1971). "The Impact of Population Growth". Science, 171(3977), pp. 1212-1217.

EHRLICH, P.R.; HOLDREN, J.P. (1972). "One-dimensional economy". Bulletin of the Atomic Scientists, 28(5), pp. 16-27.

FAN, Y.; LIU, L.C.; WU, G.; WEI, Y.M. (2006). "Analyzing impact factors of $\mathrm{CO}_{2}$ emissions using the STIRPAT model". Environmental Impact Assessment Review, 26(4), pp. 377395.

GARCÍA, J.; GARCÍA, C.B., LÓPEZ, M.M.; SALMERÓN, R. (2013). "Desarrollo del método de alzado para el tratamiento de la multicolinealidad. Determinacion del factor de alzamiento". En XXVII International Conference of Applied Economics, 1848-1862.

GARCÍA, J.; SALMERÓN, R.; GARCÍA, C.B.; LÓPEZ, M.M. (2017). "The raise estimator estimation, inference, and properties". Communications in Statistics-Theory and Methods, 46(13), pp. 6446-6462.

GASSEBNER, M.; LAMLA, M.J.; STURM, J.E. (2011). "Determinants of pollution: what do we really know?" Oxford Economic Papers, 63, pp. 568-595.

HAIR, J.F.J.; ANDERSON, R.E.; TATHAM, R.L.; BLACK, W.C. (1995). Multivariate Data Analysis. New York: MacMillan.

HARBAUGH, W.T.; LEVINSON, A.; WILSON, D.M. (2002). "Reexamining the Empirical Evidence for an Environmental Kuznets Curve." The Review of Economics and Statistics, 84(3), pp. 541-551.

JIA, J.; DENG, H.; DUAN, J.; ZHAO, J. (2009). "Analysis of the major drivers of the ecological footprint using the STIRPAT model and the PLS method. A case study in Henan Province, China." Ecological Economics, 68(11), pp. 2818-2824.

KENNEDY, P. (1992). A Guide to Econometrics. Oxford: Blackwell.

LIN, S.; ZHAO, D.; MARINOVA, D. (2009). "Analysis of the environmental impact of China based on STIRPAT model". Environmental Impact Assessment Review, 29(6), pp. 341347.

MARQUARDT, D.W. (1970). "Generalized inverses, ridge regression, biased linear estimation and nonlinear estimation." Technometrics, 12(3), pp. 591-612.

MARTíNEZ-ZARZOSO, I.; BENGOCHEA-MORANCHO, A.; MORALES-LAGE, R. (2007). "The impact of population on $\mathrm{CO} 2$ emissions: evidence from European countries." Environmental and Resource Economics, 38(4), pp. 497-512.

NETER, J.; WASSERMAN, W.; KUTNER, M.H. (1989). Applied Linear Regression Models. Homewood: Irwin.

NOVALES, A.; SALMERÓN, R.; GARCÍA, C.B.; GARCÍA, J.; LÓPEZ, M.M. (2015). "Tratamiento de la multicolinealidad aproximada mediante variables ortogonales." Anales de Economía Aplicada, pp. 1212-1227.

RASKIN, P. (1995). "Methods for estimating the population contribution to environmental change". Ecological Economics, 15(3), pp. 225-233.

ROSA, E.; DIETZ, T. (1998). "Climate change and society: speculation, construction and scientific investigation". International Sociology, 13(4), pp. 421-455. 
SALMERÓN, R.; GARCÍA, J.; GARCÍA, C.B.; GARCÍA, C. (2016). "Treatment of collinearity through orthogonal regression: an economic application". Boletín de Estadística e Investigación Operativa, 32(3), pp. 184-202.

SCHULZE, P.C. (2002). “I = PBAT”. Ecological Economics, 40(2), pp. 149-150.

UDDIN, G.; ALAM, K.; GOW, J. (2016). "Estimating the major contributors to environmental impacts in Australia". International Journal of Ecological Economics and Statistics, 37(1), pp. 1-14.

WAGGONER, P.E.; AUSUBEL, J.H. (2002). "A framework for sustainability science: a renovated IPAT identity". Proceedings of the National Academy of Sciences, 99(12), pp. 7860-7865.

YORK, R.; ROSA, E.A.; DIETZ, T. (2003). "STIRPAT, IPAT and ImPACT: analytic tools for unpacking the driving forces of environmental impacts". Ecological Economics, 46(3), pp. 351-365. 